\title{
On the axisymmetry of annular jet instabilities
}

\author{
By FALIN CHEN ${ }^{1}$, JIE-YING TSAUR ${ }^{1}$, FRANZ DURST \\ AND SAMIR K. DA $\mathbf{S}^{1}$ \\ ${ }^{1}$ Institute of Applied Mechanics, National Taiwan University, Taipei, Taiwan 106, Republic of China \\ ${ }^{2}$ Lehrstuhl fuer Stroemungsmechanik, Friedrich-Alexander-Universitaet Erlangen-Nuernberg, \\ D-91058 Erlangen, Germany
}

(Received 29 May 2002 and in revised form 18 March 2003)

We implement a linear stability analysis for the viscous annular jet surrounded by inviscid motionless gas subject to three-dimensional disturbances, and an analytical dispersion relation is presented. With this relation, we are able to obtain evidence that the axisymmetric mode is the most unstable mode of the system. The evidence for general cases is based on the numerical results obtained by solving the transcendental dispersion relation for a wide range of physical parameters. The results for two special cases, a very thin annular jet and an annular jet with disturbances of very short axial wavelength, are obtained from two rigorous analytical approaches.

\section{Introduction}

The stability of annular jets is of both fundamental and practical interest. On the practical side, the breakup of a jet into droplets due to instability has become a key technology in, for example, internal combustion engines, gas turbines, liquid rocket engines, ink-jet printers, spray coating, and so on. On the fundamental side, the stability mechanisms are influenced by, for example, the surface tension of interfaces, the flow inertia, the fluid viscosity, the thickness of annulus, and the densities of the jet and surrounding fluid. And more interestingly, the analysis can be reduced to some special cases, such as the round liquid jet, the hollow gas jet, the liquid sheet, by taking limits of relevant physical parameters. A detailed review of the research so far and a discussion on the stability characteristics can be found in, for example, Mehring \& Sirignano (2000), Chauhan et al. (2000) and Chen \& Lin (2002).

Perhaps due to the complexity of the mathematical formulation, all of the previous studies on annular jets have assumed that the instability is axisymmetric (Crapper, Dombrowski \& Pyott 1975; Meyer \& Weihs 1987; Lee \& Chen 1991; Shen \& Li 1996; Shkadov \& Sisoev 1996; Chauhan et al. 2000; Mehring \& Sirignano 2000; Chen \& Lin 2002). This assumption is valid under some circumstances, for example, a very thin annular jet of low velocity (Kendall 1986; Lee \& Wang 1986, 1989), but is not valid for others like round water jets discharging into air in particular circumstances (Lienhard 1968; Hoyt \& Taylor 1977; Stockman \& Bejan 1982; Kimura \& Bejan 1983; Villermaux 1988; Shi et al. 1999). In this paper we discard this assumption and perform a linear stability analysis for the three-dimensional disturbances on a viscous annular jet surrounded by quiescent inviscid gas. We obtain an analytical dispersion relation, with which we are able to obtain evidence that the axisymmetric mode is most unstable in the system.

The paper is organized as follows. We first derive the analytical dispersion relation in $\S 2$. We then show in $\S 3$ by a numerical approach that the axisymmetric mode is the most unstable of the system in the wide range of physical parameters considered. 


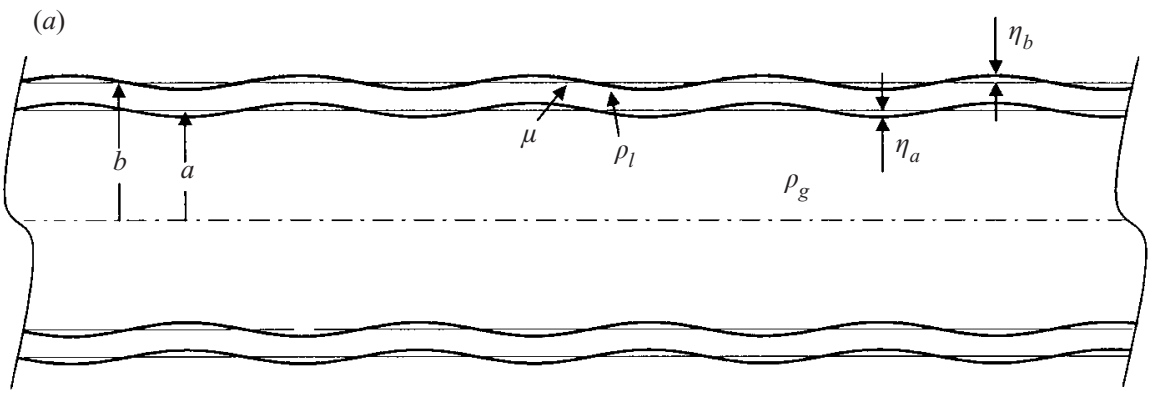

(b)

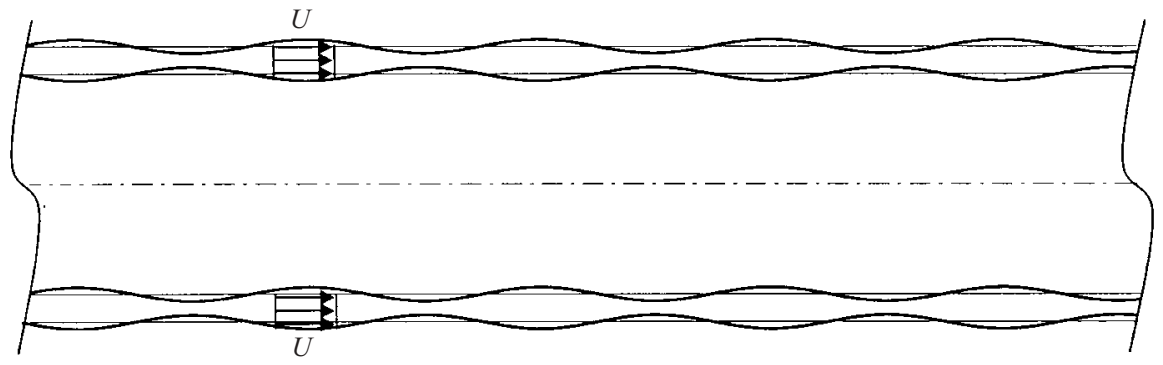

(c)

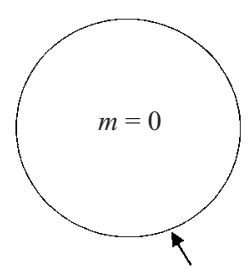

Mid-plane of non-deformed jet

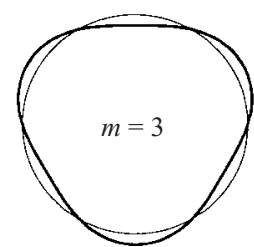

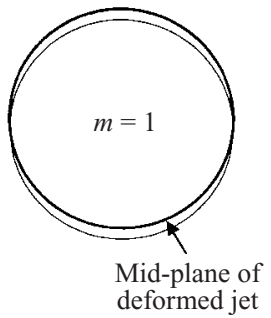

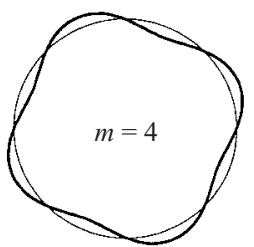

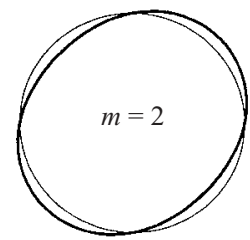

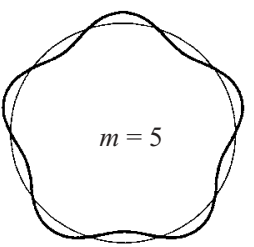

FIGURE 1. A schematic description of $(a)$ the para-sinous mode and $(b)$ the para-varicous mode. (c) The azimuthal waves of wavenumbers $m=0 ; m=1 ; m=2 ; m=3 ; m=4 ; m=5$.

We also show in $\S 4$ for two special cases by rigorous analytical approaches that the axisymmetric mode has the largest growth rate compared to other non-axisymmetric modes. Finally, in $\S 5$ concluding remarks are given.

\section{The analytical dispersion relation}

Consider an infinitely long annular liquid jet with inner radius $a$ and outer radius $b$ (figure 1). The liquid jet is of density $\rho_{l}$ and viscosity $\mu$, moving with a uniform 
axial velocity $U$ and surrounded by a motionless inviscid gas of density $\rho_{g}$ in both the inner core and outer surroundings. For such an annular jet, unstable waves develop on both the inner and outer surfaces, which may be in phase or out of phase. When the waves develop in phase, the shapes of the waves are antisymmetric with respect to the mid-plane of liquid sheet, and this kind of instability mode is called the para-sinuous mode (figure 1a). When the waves develop out of phase, the waves are symmetric with respect to the mid-plane of the liquid sheet, and this mode is called the para-varicous mode (figure 1b) (Shen \& Li 1996). Since we consider threedimensional perturbations, the two surface waves may also be axisymmetric $(m=0)$ or non-axisymmetric $(m \geqslant 1)$ with respect to the central axis of the jet (figure $1 c)$.

The governing equations of the viscous annular jet are the continuity and NavierStokes equations. In the surrounding gases both outside and inside the annulus, the flows are assumed to be inviscid and motionless. On the inner and outer surfaces of the annular jet, we have the following boundary conditions: the tangential stress vanishes, the normal stress balances the capillary force, and the kinematic condition relates the interfacial deflection to the radial velocity (Meyer \& Weihs 1987; Shen \& Li 1996). The equations of the annular jet admit a solution of uniform velocity $\boldsymbol{u}=(0,0, U)$ and zero pressure. Based on this uniform flow, we apply three-dimensional small disturbances to the annular jet and neglect the higher-order terms to render the equations linear. We then non-dimensionalize the linearized governing equations and boundary conditions by the following scales: length by $h$, velocity by $U$, time by $h / U$, and pressure by $\rho_{l} U^{2}$. After applying the normal-mode analysis and making a straightforward evaluation of the velocity field in the resultant ordinary differential equations, and relevant applications of boundary conditions, an analytical dispersion relation is obtained as follows:

$$
H_{2}\left(A \Delta_{1}-H_{1} \Delta_{3}\right)+B\left(A \Delta_{4}+H_{1} \Delta_{2}\right)=0,
$$

in which

$$
\left.\begin{array}{rl}
\Delta_{1} & =I_{m}^{\prime}(k b) K_{m}(k a)-K_{m}^{\prime}(k b) I_{m}(k a), \\
\Delta_{2} & =I_{m}^{\prime}(k a) K_{m}(k b)-K_{m}^{\prime}(k a) I_{m}(k b), \\
\Delta_{3} & =I_{m}^{\prime}(k a) K_{m}^{\prime}(k b)-K_{m}^{\prime}(k a) I_{m}^{\prime}(k b), \\
\Delta_{4} & =I_{m}(k a) K_{m}(k b)-K_{m}(k a) I_{m}(k b),
\end{array}\right\}
$$


The details of the derivation of the above dispersion relation are shown in the Appendix, in which the governing equations and boundary conditions (in linearized form) are also given. In the above and subsequent equations of the present paper, we use for briefness the notation $\left[X_{m}(k r)\right]^{\prime}=X_{m}^{\prime}(k r)$ and $\left[X_{m}(k r)\right]^{\prime \prime}=X_{m}^{\prime \prime}(k r)$, in which $X(r)$ denotes different Bessel functions and the prime and the double prime denote $\mathrm{d} / \mathrm{d} r$ and $\mathrm{d}^{2} / \mathrm{d} r^{2}$, respectively. Also, $I_{m}$ and $K_{m}$ are the modified Bessel functions of the first and second kind of order $m, k$ (real) and $m$ (integer) are the wavenumbers in respectively the axial and azimuthal directions, $R e=\rho_{l} U h / \mu$ is the Reynolds number, $W e=\sigma / \rho_{l} h U^{2}$ is the Weber number, $h$ is the characteristic length taken to be $a$ in the present paper, $\sigma$ is the surface tension, $\xi=\rho_{g} / \rho_{l}$ is the density ratio between the surrounding (or the core) gas and the liquid jet, $n=m^{2} / r^{2}+k^{2}+\operatorname{Re}(s+\mathrm{i} k)$, and $s=S_{r}+\mathrm{i} S_{i}$ is complex in which $S_{r}$ denotes the growth rate of disturbance $\left(S_{r}>0\right.$ for unstable, $S_{r}<0$ for stable).

The dispersion relations (1) and (2) are for the three-dimensional disturbances on a viscous annular jet surrounded by motionless inviscid gas, which can be put into various simplified forms for some special cases through different asymptotic approaches. For example, the simplified form for the three-dimensional inviscid annular jet can be obtained if $R e \rightarrow \infty$ is considered (Shen \& Li 1996), that for the plane liquid sheet can be obtained if $a, b \rightarrow \infty$ is considered (Li \& Tankin 1991), that for the hollow gas jet surrounded by infinite liquid can be obtained if $b \rightarrow \infty$ is considered (Li 1994), that for the round liquid jet surrounded by infinite gas can be obtained if $a \rightarrow 0$ is considered (Yang 1992), and that for the axisymmetric annular jet can be obtained if $m=0$ is considered (Shen \& Li 1996). The details of these simplification procedures are shown in Tsaur (2000).

\section{Evidence supporting axisymmetric instability: numerical approach}

The dispersion relation (1) and (2) is transcendental, and can be solved for the growth rate $S_{r}$ by a numerical approach. We did this by using MATHEMATICA, a computer code that can implement symbolic computation. In the present system, there are four relevant physical parameters: the Reynolds number Re, the Weber number $W e$, the density ratio $\xi$, and the radius ratio $b / a$. We calculate $S_{r}$ in wide ranges of these four parameters, but focus on the case: $R e=10, W e=0.0001, \xi=0.0013$ and $b / a=1.25$, which is relevant to the case of a water annular jet of medium thickness surrounded by air. We consider three values of each parameter: $R e=10,10^{3}, 10^{6}$, $W e=10^{-4}, 10^{-2}, 1, \xi=0.0013,0.01,0.1, \quad b / a=1.25,1.1,1.01$, and the results are shown respectively in figures 2 to 5 . According to previous results shown by, for example Shen \& Li (1996) and Chauhan et al. (2000), the para-sinuous mode has been found to be more unstable than the para-varicous mode in all the situations considered, which is also confirmed by the present results. We shall accordingly focus on the para-sinuous mode in the following discussion.

The numerical results on the variations of the growth rates for varying physical parameters are shown in figures 2 to 5 . From a close inspection of the numerical results, we summarize the following points regarding the axisymmetry of the instability of the system:

(i) For all the cases considered (figures 2 to 5), the maximum of $S_{r}$ of the axisymmetric mode $(m=0)$ is always larger than those of non-axisymmetric modes $(m>0)$. Namely, the axisymmetric mode is the most unstable mode in the wide parameter ranges considered. 

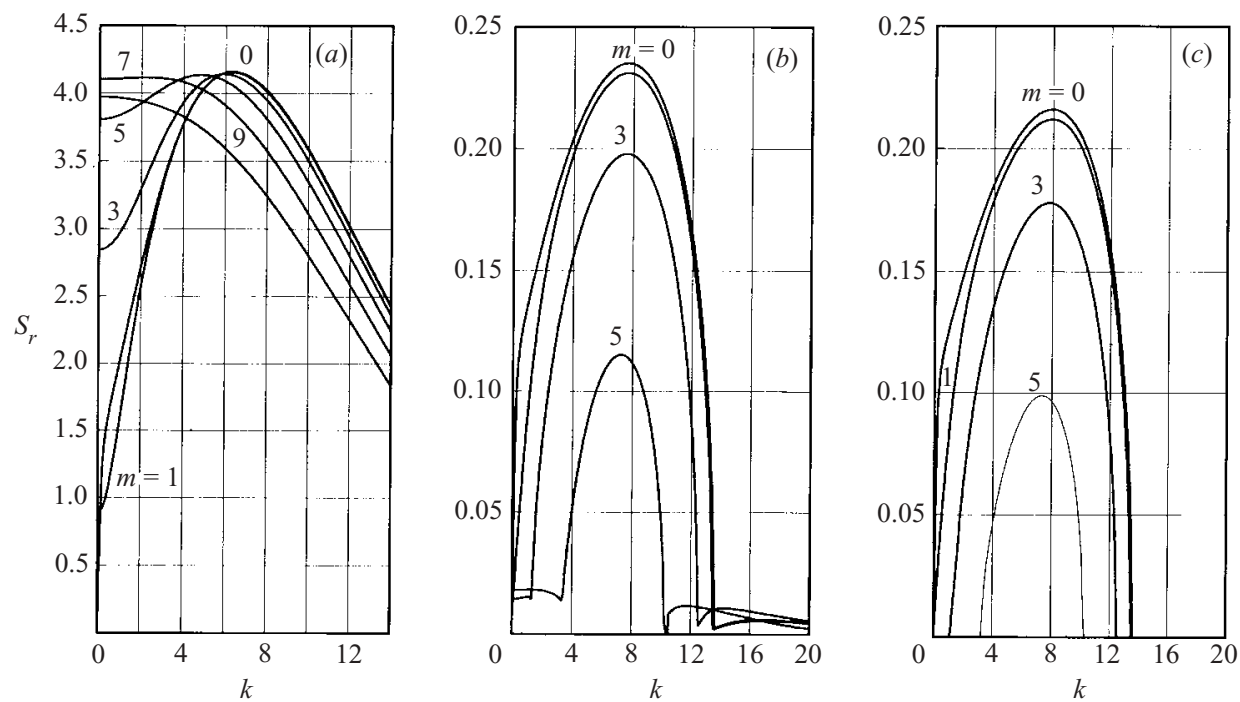

FIGURE 2. The variation of growth rate $S_{r}$ with axial wavenumber $k$ of the para-sinuous mode for $m=0$ to 9 . The case considered is $b / a=1.25, W e=0.0001$ and $\xi=0.0013$ while $R e$ varies: (a) $\operatorname{Re}=10$, (b) $\operatorname{Re}=10^{3}$ and (c) $\operatorname{Re}=10^{6}$.
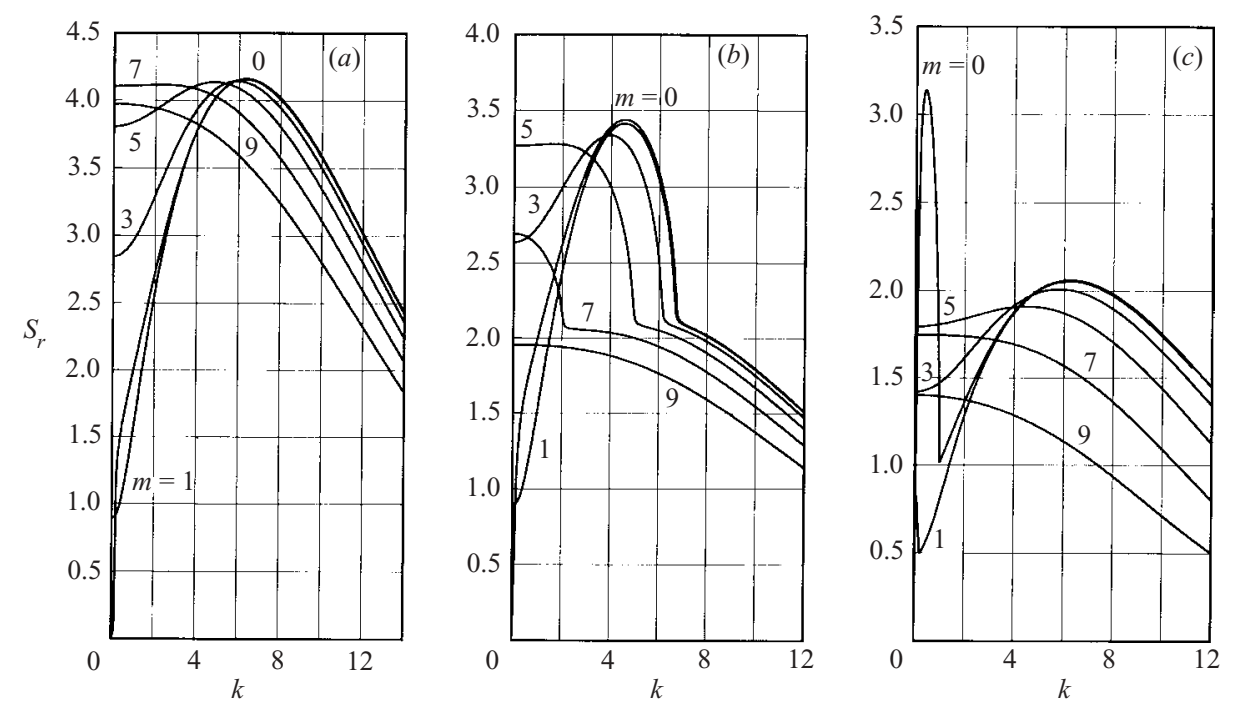

FIGURE 3. The variation of growth rate $S_{r}$ with axial wavenumber $k$ of the para-sinuous mode for $m=0$ to 9 . The case considered is $b / a=1.25, R e=10$ and $\xi=0.0013$ while $W e$ varies: (a) $W e=10^{-4},(b) W e=10^{-2}$ and (c) We $=1$.

(ii) For each case shown in each part of figures 2 to $5, S_{r}$ of the axisymmetric mode $(m=0)$ is always larger than those of non-axisymmetric modes $(m>0)$ in the regime $k \geqslant k_{c}$, where $k_{c}$ is the critical axial wavenumber at which the maximum $S_{r}$ occurs. We refer this regime of $k$ as the 'axisymmetry dominance regime'. To support this conclusion, we shall show in the next section by an analytical approach that, when the limit $k \rightarrow \infty$ is considered, $S_{r}$ of the axisymmetric mode is the largest. 

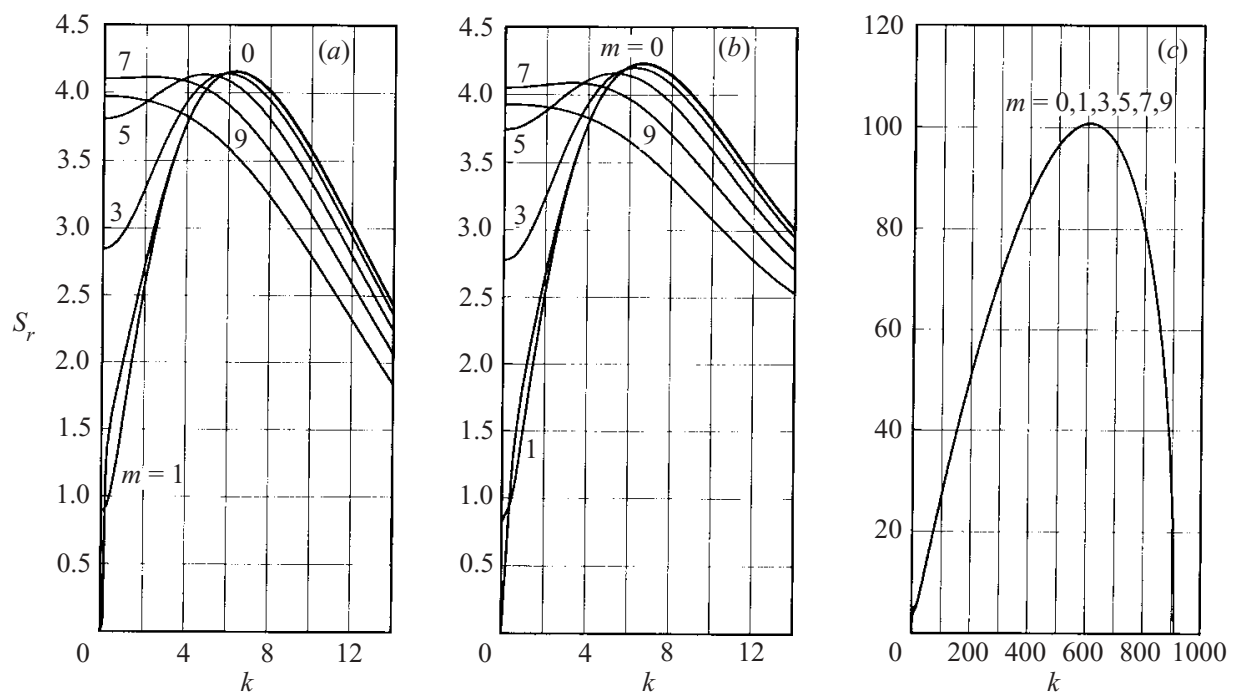

FIGURE 4. The variation of growth rate $S_{r}$ with axial wavenumber $k$ of the para-sinuous mode for $m=0$ to 9 . The case considered is $b / a=1.25, R e=10$ and $W e=0.0001$ and while $\xi$ varies: (a) $\xi=0.0013,(b) \xi=0.01$ and $(c) \xi=0.1$.
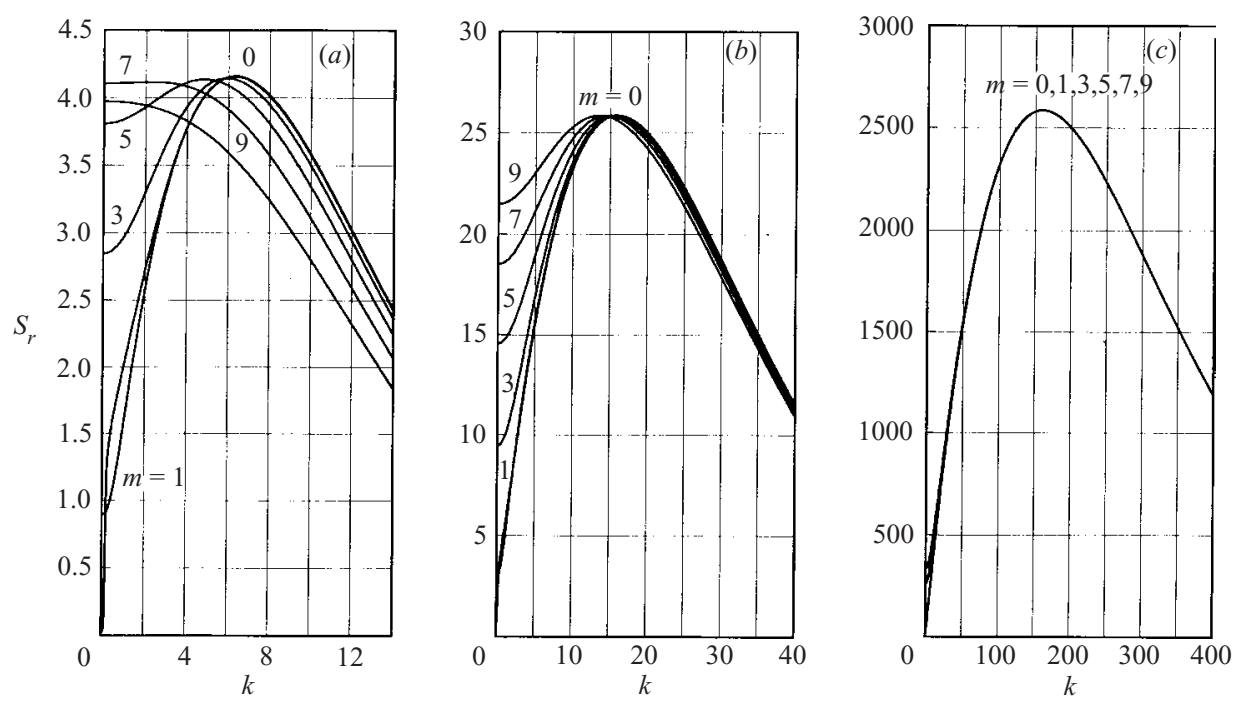

FiguRE 5. The variation of growth rate $S_{r}$ with axial wavenumber $k$ of the para-sinuous mode for $m=0$ to 9 . The case considered is $R e=10, W e=0.0001$ and $\xi=0.0013$ while $b / a$ varies: (a) $b / a=1.25$, (b) $b / a=1.1$ and $(c) b / a=1.01$.

(iii) The range of the 'axisymmetry dominance regime' is increased when some physical parameters approach a limiting value. For example, for an annular jet with a smaller density difference between jet fluid and surrounding gas $(\xi=0.1$, figure $4 c)$ or an annular jet of very thin film thickness $(b / a=1.01$, figure $5 c)$, the axisymmetry dominance regime is extended to $k \geqslant k_{l}$, where $k_{l}$ is much smaller than $k_{c}$. For a close-to-inviscid annular jet $\left(R e=10^{6}\right.$, figure $\left.2 c\right)$, the axisymmetry dominance regime has extended to the whole range of $k$ of non-zero growth rate; namely, $0 \leqslant k \leqslant k_{c o}$, 
where $k_{c o}$ is the so-called cut-off axial wavenumber beyond which the growth rate of the instability mode is zero (Cousin \& Dumouchel 1996). Again, to support this finding regarding the increase in the axisymmetry dominance regime for the limiting cases, we shall show in the next section by another analytical approach that, if the limit $b / a \rightarrow 1$ is considered, $S_{r}$ of the axisymmetric mode is again the largest (in the whole range of $k$ ).

\section{Evidence supporting axisymmetric instability: analytical approach}

A direct proof from the dispersion relation (1) and (2) that the axisymmetric mode has the largest growth rate in the whole range of physical parameters considered is an impossible task. However, the proof might be obtained for the following two limiting cases: an annular jet of very thin thickness $b / a \rightarrow 1$ and an annular jet with disturbances of very short axial wavelength $k \rightarrow \infty$. These proofs for special cases from analytical approaches can complement the numerical evidence for the general cases to support the present conclusion that the axisymmetric mode is the most unstable mode of an annular jet.

To start the analytical proof, we rewrite the dispersion relation (1) and (2) in the following form:

$$
s=f(k, m ; R e, W e, \xi, b) .
$$

We shall show that the relation $\partial s /\left.\partial m\right|_{m=0}=0$ holds, implying that the growth rate $S_{r}$ has a local maximum (not minimum, according to the numerical results shown in figures 2 to 5) when $m=0$. After some mathematical rearrangement, this relation can be written as

$$
\left.\frac{\partial s}{\partial m}\right|_{m=0}=\frac{T_{11}+T_{12}}{T_{2}},
$$

where $T_{11}, T_{12}$ and $T_{2}$ for $m=0$ are given by

$$
\begin{gathered}
T_{11}=-\frac{(s+\mathrm{i} k)}{R e}\left[2 k^{2}+(s+\mathrm{i} k) R e\right]\left(H_{2} \frac{\partial \Delta_{1}}{\partial m}+H_{1} \frac{\partial \Delta_{2}}{\partial m}\right)-\left(H_{1} H_{2} \frac{\partial \Delta_{3}}{\partial m}\right) \\
+\frac{(s+\mathrm{i} k)^{2}}{R e^{2}}\left[2 k^{2}+(s+\mathrm{i} k) R e\right]^{2} \frac{\partial \Delta_{4}}{\partial m}, \\
T_{12}=\left(B \Delta_{2}-H_{2} \Delta_{3}\right)\left[\xi s^{2} \frac{\partial}{\partial m}\left(\frac{I_{m}(k a)}{I_{m}^{\prime}(k a)}\right)-\frac{2}{R e}(s+\mathrm{i} k) \frac{\partial}{\partial m}\left(\frac{I_{m}^{\prime \prime}(k a)}{I_{m}^{\prime}(k a)}\right)\right] \\
+\left(A \Delta_{1}-H_{1} \Delta_{3}\right)\left[-\xi s^{2} \frac{\partial}{\partial m}\left(\frac{K_{m}(k b)}{K_{m}^{\prime}(k b)}\right)+\frac{2}{R e}(s+\mathrm{i} k) \frac{\partial}{\partial m}\left(\frac{K_{m}^{\prime \prime}(k b)}{K_{m}^{\prime}(k b)}\right)\right], \\
T_{2}=\frac{2\left(H_{2} \Delta_{3}-B \Delta_{2}\right)}{k I_{1}(k a)}\left(\xi s I_{0}(k a)-\frac{I_{0}^{\prime \prime}(k a)}{R e}\right)+\frac{2\left(H_{1} \Delta_{3}-A \Delta_{1}\right)}{k K_{1}(k b)}\left(\xi s K_{0}(k b)-\frac{K_{0}^{\prime \prime}(k b)}{R e}\right) \\
+\left\{\frac{(s+\mathrm{i} k)(1+R e)+2 k^{2}}{R e}\right\}\left\{\left(\Delta_{1} H_{2}+\Delta_{2} H_{1}\right)-\frac{2(s+\mathrm{i} k)}{R e}\left[2 k^{2}+(s+\mathrm{i} k) R e\right] \Delta_{4}\right\} .
\end{gathered}
$$

We then have to show that $T_{11}+T_{12}=0$ and $T_{2} \neq 0$ when $m=0$. A direct proof of (4) for a general case is mathematically intractable. We accordingly consider the following special cases, for which rigorous proofs are feasible. 


\subsection{A very thin annual jet: $b / a \rightarrow 1$}

From (5) one can see that, since

$$
\frac{(s+\mathrm{i} k)}{\operatorname{Re}}\left[2 k^{2}+(s+\mathrm{i} k) R e\right] \neq 0,
$$

$T_{11}$ will be zero if the following two conditions are satisfied at $m=0$ :

$$
\begin{gathered}
\frac{(s+\mathrm{i} k)}{\operatorname{Re}}\left[2 k^{2}+(s+\mathrm{i} k) \operatorname{Re}\right] \frac{\partial \Delta_{4}}{\partial m}-\left(H_{2} \frac{\partial \Delta_{1}}{\partial m}+H_{1} \frac{\partial \Delta_{2}}{\partial m}\right)=0, \\
\left(H_{1} H_{2} \frac{\partial \Delta_{3}}{\partial m}\right)=0 .
\end{gathered}
$$

Equations (8) and (9) can be satisfied if the following relations for $m=0$ hold:

$$
\frac{\partial \Delta_{3}}{\partial m}=\frac{\partial \Delta_{4}}{\partial m}=0, \quad H_{2} \frac{\partial \Delta_{1}}{\partial m}+H_{1} \frac{\partial \Delta_{2}}{\partial m}=0 .
$$

To show this, we consider that the thickness of the annular interface is very thin, namely $b / a \rightarrow 1$. Under this limiting condition, $\Delta_{i}(i=1,2,3,4)$ and $H_{j}(j=1,2)$ of $(2 a, b)$ can be simplified into the following forms when relevant properties of modified Bessel functions are used:

$$
\left.\begin{array}{r}
\left.\Delta_{1}\right|_{b / a \rightarrow 1}=\left[I_{m}^{\prime}(k b) K_{m}(k a)-K_{m}^{\prime}(k b) I_{m}(k a)\right]_{b / a \rightarrow 1}=1 / a, \\
\left.\Delta_{2}\right|_{b / a \rightarrow 1}=\left[I_{m}^{\prime}(k a) K_{m}(k b)-K_{m}^{\prime}(k a) I_{m}(k b)\right]_{b / a \rightarrow 1}=1 / a, \\
\left.\Delta_{3}\right|_{b / a \rightarrow 1}=\left[I_{m}^{\prime}(k a) K_{m}^{\prime}(k b)-K_{m}^{\prime}(k a) I_{m}^{\prime}(k b)\right]_{b / a \rightarrow 1}=0, \\
\left.\Delta_{4}\right|_{b / a \rightarrow 1}=\left[I_{m}(k a) K_{m}(k b)-K_{m}(k a) I_{m}(k b)\right]_{b / a \rightarrow 1}=0,
\end{array}\right\}
$$

Since $\Delta_{i}$ are constant, their derivatives with respect to $m$ will vanish. Accordingly, the two conditions of (10) are satisfied, and so are (8) and (9). Therefore, $T_{11} \equiv 0$ is proved. To show that $T_{12} \equiv 0$, we simplify (6) by using relevant relations of (11) and the fact that $A \equiv B$ when $m=0$. We then have

$$
T_{12}=A \Delta_{1}\left[\xi s^{2} \frac{\partial}{\partial m}\left(\frac{I_{m}(k a)}{I_{m}^{\prime}(k a)}-\frac{K_{m}(k a)}{K_{m}^{\prime}(k a)}\right)-\frac{2}{R e}(s+\mathrm{i} k) \frac{\partial}{\partial m}\left(\frac{I_{m}^{\prime \prime}(k a)}{I_{m}^{\prime}(k a)}-\frac{K_{m}^{\prime \prime}(k a)}{K_{m}^{\prime}(k a)}\right)\right] .
$$

It is known that when $k a \gg m$ the Bessel functions of (12) approach exponential functions, which are independent of $m$. Accordingly, both the arguments of the two derivatives on the right-hand side of (12) are independent of $m$ and, consequently, the two derivatives of (12) vanish. So that $T_{12} \equiv 0$ is confirmed.

Now, it remains to show that $T_{2} \neq 0$. We simplify (7) further by using relevant relations of (11) when $m=0$ and $b / a \rightarrow 1$ and obtain

$$
\begin{aligned}
& T_{2}=\frac{2 A}{k a}\left[-\frac{\xi s / k a}{I_{1}(k a) K_{1}(k a)}+\frac{1}{R e}\left\{\frac{I_{0}^{\prime \prime}(k a)}{I_{1}(k a)}+\frac{K_{0}^{\prime \prime}(k a)}{K_{1}(k a)}\right\}\right] \\
& +\left\{\frac{(s+\mathrm{i} k)(1+R e)+2 k^{2}}{\operatorname{Re}}\right\} \frac{1}{a}\left(H_{1}+H_{2}\right)_{b / a \rightarrow 1} .
\end{aligned}
$$


Since $\left(H_{1}+H_{2}\right)_{b / a \rightarrow 1} \neq 0$ and the terms involving $I_{0}(k a), K_{0}(k a)$ and their firstand second-order derivatives are all non-zero for finite $k a, T_{2} \neq 0$ must hold. In conclusion, we have shown that, for a very thin annular jet, i.e. $b / a \rightarrow 1$, the relation $\partial s /\left.\partial m\right|_{m=0}=0$ holds. Namely, the axisymmetric instability mode has the largest growth rate, and is therefore the most unstable mode and will dominate the instability of the system.

This proof is supported by the numerical results for the case $b / a=1.01$ (figure $2 c$ ) in which the growth rate of the axisymmetric mode in virtually the whole range of $k$ considered $\left(25 \approx k_{l} \leqslant k \leqslant k_{c o} \approx 700\right)$ is invariably larger than those of non-axisymmetric modes. This proof is also supported by the results of Kendall (1986) and Lee \& Wang (1986, 1989), who showed that for a thin annular jet with a slow jet flow, the shape of the annular jet was always observed to be axisymmetric.

\subsection{An annular jet with disturbances of very short axial wavelength: $k \rightarrow \infty$}

In this subsection, we will show that for an annular jet of finite thickness $(a \neq b)$ with disturbances of very short axial wavelength $(k \rightarrow \infty)$ the axisymmetric mode has the largest growth rate. We consider the limit $k \rightarrow \infty$ and use the limiting forms of modified Bessel functions $I_{m}$ and $K_{m}$ as $k \rightarrow \infty$; then $\Delta_{i}(i=1,2,3,4)$ and $H_{j}(j=1,2)$ can be reduced to the following when $k a \gg m$ :

$$
\begin{gathered}
\Delta_{1} \rightarrow \frac{1}{2 \sqrt{a b}}\left(\frac{\mathrm{e}^{k b}}{\mathrm{e}^{k a}}+\frac{\mathrm{e}^{k a}}{\mathrm{e}^{k b}}\right), \quad \Delta_{2} \rightarrow \frac{1}{2 \sqrt{a b}}\left(\frac{\mathrm{e}^{k b}}{\mathrm{e}^{k a}}+\frac{\mathrm{e}^{k a}}{\mathrm{e}^{k b}}\right), \\
\Delta_{3} \rightarrow \frac{k}{2 \sqrt{a b}}\left(\frac{\mathrm{e}^{k b}}{\mathrm{e}^{k a}}-\frac{\mathrm{e}^{k a}}{\mathrm{e}^{k b}}\right), \quad \Delta_{4} \rightarrow \frac{1}{2 k \sqrt{a b}}\left(\frac{\mathrm{e}^{k a}}{\mathrm{e}^{k b}}-\frac{\mathrm{e}^{k b}}{\mathrm{e}^{k a}}\right), \\
H_{1} \rightarrow \frac{\xi}{k} s^{2}-W e\left(\frac{1}{a^{2}}-k^{2}\right)-\frac{2 k}{R e}(s+\mathrm{i} k), \\
H_{2} \rightarrow \frac{\xi}{k} s^{2}-W e\left(\frac{1}{b^{2}}-k^{2}\right)-\frac{2 k}{R e}(s+\mathrm{i} k), \\
\left.A\right|_{k \rightarrow \infty}=\left.B\right|_{k \rightarrow \infty}=-\frac{(s+\mathrm{i} k)}{R e}\left[2 k^{2}+\operatorname{Re}(s+\mathrm{i} k)\right] .
\end{gathered}
$$

Note that in $(14 a)$ the $\Delta_{i}$ are, again, not function of $m$, and therefore their derivatives with respect to $m$ will vanish. From a close inspection of the order of magnitude of the terms of (14) we found from order analyses that $\Delta_{1} \equiv O\left(\mathrm{e}^{k(b-a)}\right)$, $\Delta_{2} \equiv O\left(\mathrm{e}^{k(b-a)}\right), \Delta_{3} \equiv O\left(k \mathrm{e}^{k(b-a)}\right), \Delta_{4} \equiv O\left(\mathrm{e}^{k(b-a)} / k\right), H_{1} \equiv O\left(k^{2}\right)$ and $H_{2} \equiv O\left(k^{2}\right)$ when $m=0$. Accordingly, the highest order of $k$ of $T_{11}$ is $O\left(k^{6}\right)$, that of $T_{12}$ is $O\left(k^{3} \mathrm{e}^{k(b-a)}\right)$, and that of $T_{2}$ is $O\left(k^{4} \mathrm{e}^{k(b-a)}\right)$. By dividing each term by $k^{4} \mathrm{e}^{k(b-a)}$ and taking the limit as $k \rightarrow \infty$, we conclude that $T_{11} \rightarrow 0$ after applying L'Hopital's rule twice. Similarly, $T_{12} \rightarrow 0$ and $T_{2} \rightarrow O(1) \neq 0$ can be also obtained by a similar order analysis. In conclusion, when the axial wavelength of the disturbance is very small $(k \rightarrow \infty)$, the axisymmetric mode has the largest growth rate. This analytical proof is supported by all the numerical results shown in figures 2 to 5 that as $k>k_{c}$ the value of $S_{r}$ for the axisymmetric mode is always larger than those of non-axisymmetric modes.

\section{Concluding remarks}

We have implemented a linear stability analysis for three-dimensional small disturbances on an annular jet surrounded by motionless inviscid gas. Through a formal mathematical operation, we obtain a dispersion relation in analytical form, (1) 
and (2). This general relation is useful because it can be reduced to special relations for some limiting cases by taking an asymptotic approach for relevant physical parameters; examples are the three-dimensional inviscid annular jet if $R e \rightarrow \infty$, the plane liquid sheet if $a, b \rightarrow \infty$, the hollow gas jet surrounded by infinite liquid if $b \rightarrow \infty$, the round liquid jet surrounded by infinite gas if $a \rightarrow 0$, and the axisymmetric annular jet if $m=0$. Moreover, using this relation, the stability characteristics of an annular jet under some special situations, such as in the extremely long-axialwavelength regime $(k \rightarrow 0)$, in the extremely short-axial-wavelength regime $(k \rightarrow \infty)$, an extremely thin annular jet $(b / a \rightarrow 1)$, can also be examined. In summary, the dispersion relation can be very useful for a stability analysis of cases related to the annular jet, through which we can obtain complete information regarding the stability of the jet system considered.

More significantly, with the aid of this analytical dispersion relation, we are able to show analytically that, for two special jets, a very thin annular jet $(b / a \rightarrow 1)$ and an annular jet with disturbances of very small axial wavelength $(k \rightarrow \infty)$, the axisymmetric instability mode has the largest growth rate. For annular jets of arbitrary form, our numerical results calculated from (1) and (2) for a wide range of physical parameters also indicate that the axisymmetric mode is the most unstable one. The numerical results also support the analytical proofs for the limiting cases considered. This conclusion improves on all previous investigations of the stability of annular jets, in which only the axisymmetric mode has been considered.

To date, to the best of our knowledge, only investigations of the stability of the round jet have considered the possible onset of non-axisymmetric modes (for example Yang 1992; Lin \& Webb 1994; Avital 1995; Parthasarathy \& Chiang 1998), whereas linear stability results indicated invariably that the axisymmetric mode always has the largest growth rate. Experimental works (for example, Lienhard 1968; Hoyt \& Taylor 1977; Stockman \& Bejan 1982; Kimura \& Bejan 1983; Villermaux 1988; Shi et al. 1999) have reported that the non-axisymmetric modes might occur, but only in the nonlinear regime. In the present paper we show that, for the physical parameter regimes considered and for the limiting cases considered, the axisymmetric mode is the most unstable one. Although we are not able to show that this conclusion will invariably hold under any circumstances, we nevertheless, based on the present and previous results, would expect this to be the case.

We note that Chen \& Lin (2002) studied an annular jet in a pipe, including consideration of the viscosity of the surrounding gas; again, only the axisymmetric mode was considered. In such a system, there are 10 physical parameters influencing the stability characteristics, and the eigenvalue problem must be solved by a numerical approach, which, however, required a very close initial guess to obtain a convergent solution. In our experience, due to the large parameter space as well as the strict limitation on the initial guess, it would be very difficult to implement a threedimensional stability analysis of such a system through the numerical approach, let alone an analytical approach as the present paper shows. Nevertheless, whether the effects due to the motion of a surrounding viscous gas would influence the conclusion of present paper merits future research.

F. C. expreses his grateful appreciation to Alexander von Humboldt foundation of Germany for providing a fellowship for him to pursue a one-year research stay in the University of Erlangen to initiate this work. The work was finished in Taiwan under the financial support from National Science Council grant NSC 88-2212-E-002-018. 
Appendix. The derivation of dispersion relation (1) and (2)

Consider the infinitely long annular liquid jet with inner radius $a$ and outer radius $b$ shown in figure 1. A three-dimensional linear stability analysis on the jet is performed. The linearized small-perturbation equations and boundary conditions are obtained through routine linear stability analysis procedures. After applying a normal-mode expansion, we obtain the following ordinary differential equations:

$$
\begin{gathered}
\frac{\hat{u}_{r}}{r}+\hat{u}_{r}^{\prime}+\mathrm{i}\left(\frac{m}{r} \hat{u}_{\theta}+k \hat{u}_{z}\right)=0, \\
\hat{u}_{r}^{\prime \prime}+\frac{1}{r} \hat{u}_{r}^{\prime}-\left[\frac{1+m^{2}}{r^{2}}+k^{2}+R e(s+\mathrm{i} k)\right] \hat{u}_{r}-\hat{p}^{\prime} R e-\frac{2 \mathrm{i} m}{r^{2}} \hat{u}_{\theta}=0, \\
\hat{u}_{\theta}^{\prime \prime}+\frac{1}{r} \hat{u}_{\theta}^{\prime}-\left[\frac{1+m^{2}}{r^{2}}+k^{2}+\operatorname{Re}(s+\mathrm{i} k)\right] \hat{u}_{\theta}-\frac{\mathrm{i} m}{r} \hat{p} R e+\frac{2 \mathrm{i} m}{r^{2}} \hat{u}_{r}=0, \\
\hat{u}_{z}^{\prime \prime}+\frac{1}{r} \hat{u}_{z}^{\prime}-\left[\frac{m^{2}}{r^{2}}+k^{2}+\operatorname{Re}(s+\mathrm{i} k)\right] \hat{u}_{z}-\mathrm{i} k \hat{p} R e=0 .
\end{gathered}
$$

Similarly, the kinematic conditions at $r=a^{+}$and $r=b^{-}$are obtained as

$$
\begin{aligned}
& \left.\hat{u}_{r}\right|_{r=a^{+}}=s \varepsilon_{a}=\left.\frac{s}{s+\mathrm{i} k} \hat{u}_{r}\right|_{r=a^{+}}, \\
& \left.\hat{u}_{r}\right|_{r=b^{-}}=s \varepsilon_{b}=\left.\frac{s}{s+\mathrm{i} k} \hat{u}_{r}\right|_{r=b^{-}},
\end{aligned}
$$

the dynamic conditions on $r=a^{+}$and $r=b^{-}$as

$$
\begin{gathered}
\mathrm{i} k \hat{u}_{r}+\hat{u}_{z}^{\prime}=0, \\
\hat{u}_{\theta}^{\prime}-\frac{\hat{u}_{\theta}}{r}+\frac{\mathrm{i} m}{r} \hat{u}_{r}=0,
\end{gathered}
$$

and the pressure-continuity conditions as

$$
\begin{aligned}
& -\xi \hat{p}_{a}=-\hat{p}+W e \varepsilon_{a}\left(\frac{1-m^{2}}{a^{2}}-k^{2}\right)+\frac{2}{R e} \hat{u}_{r}^{\prime} \text { at } r=a^{+}, \\
& -\xi \hat{p}_{b}=-\hat{p}-W e \varepsilon_{b}\left(\frac{1-m^{2}}{b^{2}}-k^{2}\right)+\frac{2}{R e} \hat{u}_{r}^{\prime} \text { at } \quad r=b^{-} .
\end{aligned}
$$

We note that

$$
\nabla^{2} p=0,
$$

which is obtained by taking the divergence of the Navier-Stokes equation and applying continuity to eliminate the velocity terms. By applying a normal-mode expansion to (A 11), we have

$$
\hat{p}^{\prime \prime}+\frac{1}{r} \hat{p}^{\prime}-\left(\frac{m^{2}}{r^{2}}+k^{2}\right) \hat{p}=0 .
$$

It is seen that (A 12) is a Bessel equation; its solution can be written as the combination of modified Bessel functions of order $m$. Namely, for the liquid jet the solution is

$$
\hat{p}(r)=A_{1} I_{m}(k r)+B_{1} K_{m}(k r) \text { in } a \leqslant r \leqslant b .
$$

For the surrounding gas, the pressure is bounded, so that

$$
\begin{aligned}
& \hat{p}_{a}(r)=A_{2} I_{m}(k r) \quad \text { in } \quad r \leqslant a, \\
& \hat{p}_{b}(r)=B_{2} K_{m}(k r) \quad \text { in } \quad r \geqslant b .
\end{aligned}
$$


In (A 13), (A 14) and (A 15) there are four coefficients $A_{1}, B_{1}, A_{2}$, and $B_{2}$ to be determined, so that four conditions on pressure are required. Equations (A 9) and (A 10) will be utilized first, and the terms containing $\hat{u}_{r}^{\prime}$ will be considered initially. We exploit the momentum equations of the surrounding gas and the kinematic conditions (A 5) and (A 6) and obtain

$$
\begin{aligned}
& \hat{p}_{a}^{\prime}(r)=-\frac{s^{2}}{s+\mathrm{i} k} \hat{u}_{r} \quad \text { at } \quad r=a^{+}, \\
& \hat{p}_{b}^{\prime}(r)=-\frac{s^{2}}{s+\mathrm{i} k} \hat{u}_{r} \quad \text { at } \quad r=b^{-} .
\end{aligned}
$$

We then differentiate (A 14) and (A 15) and substitute the results into (A 16) and (A 17) and obtain

$$
\begin{aligned}
& \hat{u}_{r}=-\frac{s+\mathrm{i} k}{s^{2}} I_{m}^{\prime}(k a) A_{2} \quad \text { at } \quad r=a^{+}, \\
& \hat{u}_{r}=-\frac{s+\mathrm{i} k}{s^{2}} K_{m}^{\prime}(k b) B_{2} \quad \text { at } \quad r=b^{-} .
\end{aligned}
$$

We denote $\mathrm{d} I_{m}(k r) /\left.\mathrm{d} r\right|_{r=a}$ by $I_{m}^{\prime}(k a)$ and correspondingly for $K_{m}(k r)$. Consequently, (A 9), (A 10) and (A 18) and (A 19) consist of two of the four conditions required to solve the four coefficients in (A 13) to (A 15). To obtain another two conditions, we seek another relation between $\hat{u}_{r}$ and $\hat{p}$ by eliminating $\hat{u}_{\theta}$ in (A 2) as relevant boundary conditions are applied, resulting in

$$
\left[\frac{m^{2}}{r^{2}}+k^{2}+n-\frac{\left(2 m^{2} / r^{2}\right)\left(2 k^{2}+n\right)}{n\left(k^{2} r^{2}+m^{2}\right)}\right] \hat{u}_{r}-\frac{2 m^{2} / r}{k^{2} r^{2}+m^{2}} \hat{u}_{r}^{\prime}+\hat{p}^{\prime} R e=0,
$$

in which $n=m^{2} / r^{2}+k^{2}+\operatorname{Re}(s+\mathrm{i} k$ ). Equation (A 20) holds for both $r=a$ and $r=b$, and these are the two equations we are seeking. So the four coefficients $A_{1}, B_{1}$, $A_{2}$ and $B_{2}$ can be determined by the four homogeneous equations (A 9), (A 10) and (A 20) for $r=a$ and $b$. The dispersion relation (1) and (2) can therefore be obtained by the $4 \times 4$ determinant since these four coefficients cannot be zero simultaneously.

\section{REFERENCES}

Avital, E. 1995 Asymmetric instability of a viscid capillary jet in an inviscid media. Phys. Fluids 7, $1162-1164$.

Chauhan, A., Maldarelli, C., Papageorgiou, D. T. \& Rumschitzki, D. S. 2000 Temporal instability of compound threads and jet. J. Fluid Mech. 420, 1-25.

Chen, J. N. \& LIN, S. P. 2002 Instability of an annular jet surrounded by a viscous gas in a pipe. J. Fluid Mech. 450, 235-258.

Cousin, J. \& Dumouchel, C. 1996 Effect of viscosity on the linear instability of a flat liquid sheet. Atomization and Sprays 6, 563-576.

Crapper, G. D., Dombrowski, N. \& PyotT, G. A. 1975 Kelvin-Helmholtz wave growth on cylindrical sheets. J. Fluid Mech. 68, 497-502.

Hyot, J. W. \& TAYlor, J. J. 1977 Waves on water jets. J. Fluid Mech. 83, 119-127.

Kendall, J. K. 1986 Experiments on annular liquid jet instability and on the formation of liquid shells. Phys. Fluids 29, 2086-2094.

Kimura, S. \& BeJan, A. 1983 The buckling of a vertical liquid column. Trans. ASME: J. Fluids Engng 105, 469-473.

LeE, C. P. \& Wang, T. G. 1986 A theoretical model for the annular jet instability. Phys. Fluids 29, 2076-2085.

LeE, C. P. \& Wang, T. G. 1989 The theoretical model for the annular jet instability - revisited. Phys. Fluids A 1, 967-974. 
LeE, J.-G. \& ChEN, L.-D. 1991 Linear stability analysis of gas-liquid interface. AIAA J. 29, 15891595.

LI, X. 1994 On the instability of plane liquid sheets in two gas streams of unequal velocities. Acta Mechanica 106, 137-156.

LI, X. \& Tankin, R. S. 1991 On the temporal instability of a two-dimensional viscous liquid sheet. J. Fluid Mech. 226, 425-443.

Lienhard, J. H. 1968 Capillary action in small jets impinging on liquid surfaces. Trans. ASME: J. Basic Engng 90, 137-138.

Lin, S. P. \& WebB, R. 1994 Nonaxisymmetric evanescent waves in a viscous liquid jet. Phys. Fluids 6, 2545-2547.

Mehring, C. \& Sirignano, W. A. 2000 Axisymmetric capillary waves on thin annular liquid sheets. I. Temporal stability. Phys. Fluids 12, 1417-1439.

Meyer, J. \& Weins, D. 1987 Capillary instability of an annular liquid jet. J. Fluid Mech. 179, 531-545.

Parthasarathy, R. N. \& Chiang, K.-M. 1998 Temporal instability of gas jets injected in viscous liquids to three-dimensional disturbances. Phys. Fluids 10, 2105-2107.

Shen, J. \& LI, X. 1996 Instability of an annular viscous liquid jet. Acta Mechanica 114, 167-183.

SHI, S. X., XI, D. G., QIN, J. R., LiU, N. \& SHU, G. C. 1999 Unstable asymmetric modes of a liquid jet. Trans. ASME: J. Fluids Engng 121, 379-383.

Shkadov, V. Ya. \& Sisoev, G. M. 1996 Instability of a two-layer capillary jet. Intl J. Multiphase Flow 22, 363-377.

Stockman, M. G. \& Bejan, A. 1982 The nonaxisymmetric (buckling) flow regime of fast capillary jets. Phys. Fluids 25, 1506-1511.

Tsaur, J. Y. 2000 Stability analysis of the annular jet. Master thesis, National Taiwan University, Taipei, Taiwan.

Villermaux, E. 1998 Mixing and spray formation in coaxial jets. J. Propul. Power 14, 807-817.

YANG, H. Q. 1992 Asymmetric instability of a liquid jet. Phys. Fluids A 4, 681-689 